\title{
MoleculARweb: a website for chemistry and structural biology education through interactive augmented reality out of the box in commodity devices
}

Fabio Cortés Rodríguez, ${ }^{1}$ Gianfranco Frattini, ${ }^{2}$ Lucien F. Krapp, ${ }_{1}^{1}$ Hassan Martinez-Huang, ${ }^{3}$ Diego M. Moreno, ${ }^{2,4}$ Jorge Salomón, ${ }^{5}$ Lee Stemkoski, ${ }^{6}$ Sylvain Traeger, ${ }^{1}$ Matteo Dal Peraro ${ }^{1}$ and Luciano A. Abriata $^{1 *}$

${ }^{1}$ Laboratory for Biomolecular Modeling, École Polytechnique Fédérale de Lausanne and Swiss Institute of Bioinformatics, 1015 Lausanne, Switzerland; ${ }^{2}$ Área Química General e Inorgánica, Departamento de Química-Física, Facultad de Ciencias Bioquímicas y Farmacéuticas, Universidad Nacional de Rosario, Suipacha 531, S2002LRK Rosario, Santa Fe, Argentina; ${ }^{3}$ Universidad de Oriente, Avenida Patricio Lumumba S/N, 90500 Santiago de Cuba, Cuba; ${ }^{4}$ Instituto de Química de Rosario (CONICET-UNR), Suipacha 570, S2002LRK Rosario, Santa Fe, Argentina; ${ }^{5}$ Escuela de Educación Técnica Nro. 4, I Brigada Aérea, el Palomar, Argentina; ${ }^{6}$ Adelphi University, New York 11530, U.S.A.

* To whom correspondence should be addressed: luciano.abriata@epfl.ch

\begin{abstract}
Augmented reality (AR) technologies hold the promise to revolutionize how students learn about molecular structure in chemistry and biology. However, the AR tools easier to implement in the classroom are largely limited to static visualizations without any interactivity, while more interactive solutions require ad hoc hardware -some very expensive, others affordable but still far from commodity to enable widespread use- and complex, in some cases even paid, software.

Here we introduce MoleculARweb, a purely web-based AR platform for chemistry, molecular and structural biology education that runs out of the box in regular web browsers in ordinary computers, laptops, tablets and smartphones. Through regular-printed markers recognized via the device's webcam, students and teachers can handle molecules in an AR environment to interactively explore their structure, dynamics and interactions. The release version of MoleculARweb features over 20 activities covering topics about molecular shapes, atomic and molecular orbitals, acid-base equilibria and hydrogen bonding, protein and nucleic acid structures, X-ray diffraction and electron microscopy, and molecular mechanics; applicable from high school to early university levels. MoleculARweb is freely available without registration at https://MoleculARweb.epfl.ch in English, Spanish, French, Italian, German and Portuguese languages.

A qualitative assessment on high-school and undergraduate students, and in science communication outreach activities for the public, showed strong engagement and suggested some positive effects on learning. Having MoleculARweb available online for widespread use, we next plan to collect large-scale statistics to quantitatively mass-test the actual utility and effects of AR technologies in chemistry and biology education.
\end{abstract}




\section{Introduction}

Computer graphics applications for molecular visualization are well-established tools for teaching and self-learning about molecular structures, properties and reactivity in chemistry and biology courses. $^{1-3}$ An important limitation of regular computer graphics programs is the unintuitive link between the six degrees of freedom naturally available to each of the user's limbs, the singlehanded 2D nature of mouse-driven operations, and the 3D nature of molecular structures and interactions between pairs of molecules. Human interaction with molecular models is in fact much more fluid with physical molecular modeling (such as plastic-made kits for small molecules and proteins, 3D-printed models ${ }^{4,5}$, etc.) than with $2 \mathrm{D}$ graphics and a mouse, because the tangible model enables seamless manipulation of the 3D structure and even of its internal flexibility. The user can also more easily compare two tangible molecules by moving them independently with the hands, than two molecules handled with a single mouse on a flat screen. On the other hand, physical kits for molecular modeling have themselves several limitations including restricted number and types of atoms available, the need to manually build each model, and the lack of any augmentation that computers offer, such as colors, textures and labels to display surfaces, measurements, or auxiliary information. Besides, physical models are limited to atom representations of molecules and at most secondary structure representations of proteins. Some studies suggest that combining computer graphics and physical models may help to better grasp 3D structures compared to using any of them alone. ${ }^{6}$ Here we report a large set of activities that intend to blend physical and virtual experiences for chemistry and biology education, through augmented reality in a format delivered directly through web browsers and operational without any specific hardware or software.

Technologies for advanced human-computer interaction using augmented and virtual reality (AR/VR) aim at connecting the physical space around users with simulated objects that they can handle in space. In VR the experience is fully virtual, including a virtual environment, while in AR the users see the virtual objects overlaid onto the physical world. The main advantage of AR/VR tools over regular computer graphics relies in the full 3D control of the visualization, even if actually displayed on a flat screen, which allows a much more natural interaction with the molecules. ${ }^{7}$ Moreover, AR/VR applications typically enable the independent use of two hands to manipulate multiple virtual objects simultaneously. AR and VR thus allow for much more complex operations than standard computer graphics, which has been shown to improve manipulation of virtual objects including virtual molecules. ${ }^{8} \mathrm{AR}$ is of special interest, as it keeps the user in view of its limbs thus further facilitating coordination compared to $\mathrm{VR},{ }^{9}$ and of the surroundings thus not disrupting integration with persons around, important for students in a class.

Chemistry and structural biology have been particularly fruitful niches for AR/VR, with a rapid growth in the number of software tools that implement such technologies both for education and for actual research. ${ }^{10-26}$ Despite all the purported advantages of AR and VR for molecular modeling in education, several important issues stand. First, most tools for handling molecules in $\mathrm{AR} / \mathrm{VR}$ require $\mathrm{ad}$ hoc hardware that is expensive despite the dropping costs, precluding one-device-per-student adoption even in wealthy countries. Second, software setups are often complex for lay users, while the simpler alternatives offer no more than visualization capabilities; moreover, many of these programs are paid. Third, because these issues strongly limit availability and usability, we lack large-scale testing about the actual pedagogical effect of AR/VR tools over conventional ones, usually attributed large potential in assisting thinking and learning processes in scientific disciplines that involve abstract, intangible, idealized concepts but never thoroughly proved. ${ }^{27,28}$ Last, most of the pedagogical tools with modest hardware requirements that could hence become widely used, are quite limited to the display of static graphics, certainly insightful but with no interactivity nor any kind of molecular mechanics.

We recently described how client-side web programming ${ }^{29,30}$ can offer a relatively simple way to encode interactive molecular graphics applications into AR experiences without the need of specialized hardware and running directly inside web browsers on regular computers. ${ }^{26}$ Here we introduce the first formal release of MoleculARweb, a website that contains a large number of activities for interactive AR-based molecular visualization running in regular web browsers on ordinary computers, laptops, tablets and smartphones equipped with a webcam and internet connection. Users only need to print 2D markers that are then recognized via webcam allowing them to handle molecules in an AR environment to interactively explore their structure, dynamics and interactions. Activities available by the time of first release cover topics about molecular shapes, electronic orbitals, acid-base equilibria and hydrogen bonding, structures of biological macromolecules, X-ray diffraction and electron microscopy, and molecular mechanics. One of the main activities is a prototype of an AR-based 
molecular modeling kit where users can build any model and explore its structure and dynamics akin to a plastic-made model.

The website is free to use without registration at https://MoleculARweb.epfl.ch, including at the moment over 20 activities available in English, Spanish, Italian, French, Portuguese and German languages. Preliminary tests in school visits and in public outreach activities showed good engagement from students and the public. Teachers and students are encouraged to try these activities and to optionally fill in the surveys, which will help us evaluate at large scale the pedagogical utility of the website and AR technologies, as well as guide the development of further content.

\section{Overall description of the website}

Briefly, our website uses free, open JavaScript implementations of computer vision and graphics libraries to capture AR markers printed on regular paper with a standard printer (Figure 1A and Figures $\mathrm{S} 1$ and S2 with markers ready to print in different sizes) through the webcam, and to overlay on top of them the atoms, molecules or any other kind of virtual object as needed to render each experience. ${ }^{26}$ Once an activity is selected from the main menu (Figure 1B), the user moves and rotates the AR markers in front of the webcam to drive the position and orientation of the virtual objects (Figure 1C) and thus trigger interactivity for the interactive examples. For best experiences we recommend using the website in a regular laptop or computer that acts as a mirror showing back the user holding the molecules (Figure 1C) or in look-through mode on smartphones and tablets with the back webcam (Figure 1D). Modern smartphones further support marker-less visualizations that stand on flat surfaces in $A R$ (Figure 1E), that the website makes available for visualization-only activities. The figures in this article exemplify marker-based and marker-less uses.

The website has been verified to work in all AR modes on several smartphones and laptop computers with Windows, Linux, Mac and Android operating systems, in Chrome, Firefox, Edge and Safari web browsers. We recommend Chrome as the best-tested browser, in any operating system. To use the different activities described in the next section, the user needs to access the webpage of interest, enable the webcam when the browser prompts, show the AR marker(s) indicated to the webcam and follow the instructions. Most activities employ the flat two-sided markers labeled 1 and 2 in Figure 1A (Figure S1) which need to be folded back to back and glued. Attaching a small stick as in the figures helps to control the marker without hiding any part of it from the webcam. One activity employs the cube marker of Figure 1A (printable from Figure S2), also better if folded and glued to a solid cube. Table S1 lists potential problems and solutions; among the key ones are ensuring https URL (not http), enabling the webcam when the web browser prompts, leaving a white space around the printed AR markers, and having good lighting conditions but not direct strong light on the AR markers.

Users can directly access all activities from the main screen (Figure 1B) which also holds general instructions, links to relevant papers and additional information. Here or in each activity, users can get the required markers, chose language, etc. The screens for activities further contain general controls to zoom, swap video and graphics, etc. (Figure 1D) plus activity-specific controls that enable choosing among systems and provide interactivity. Note that besides the marker-based handling of virtual objects, most activities further support zooming and rotations with standard mouse operations or with fingers in touchenabled devices when the rotation mode is on. This enables the incorporation of fixed markers into documents onto which the apps can directly display the virtual objects, and that multiple users employing the same AR marker can independently rotate the object. 
A

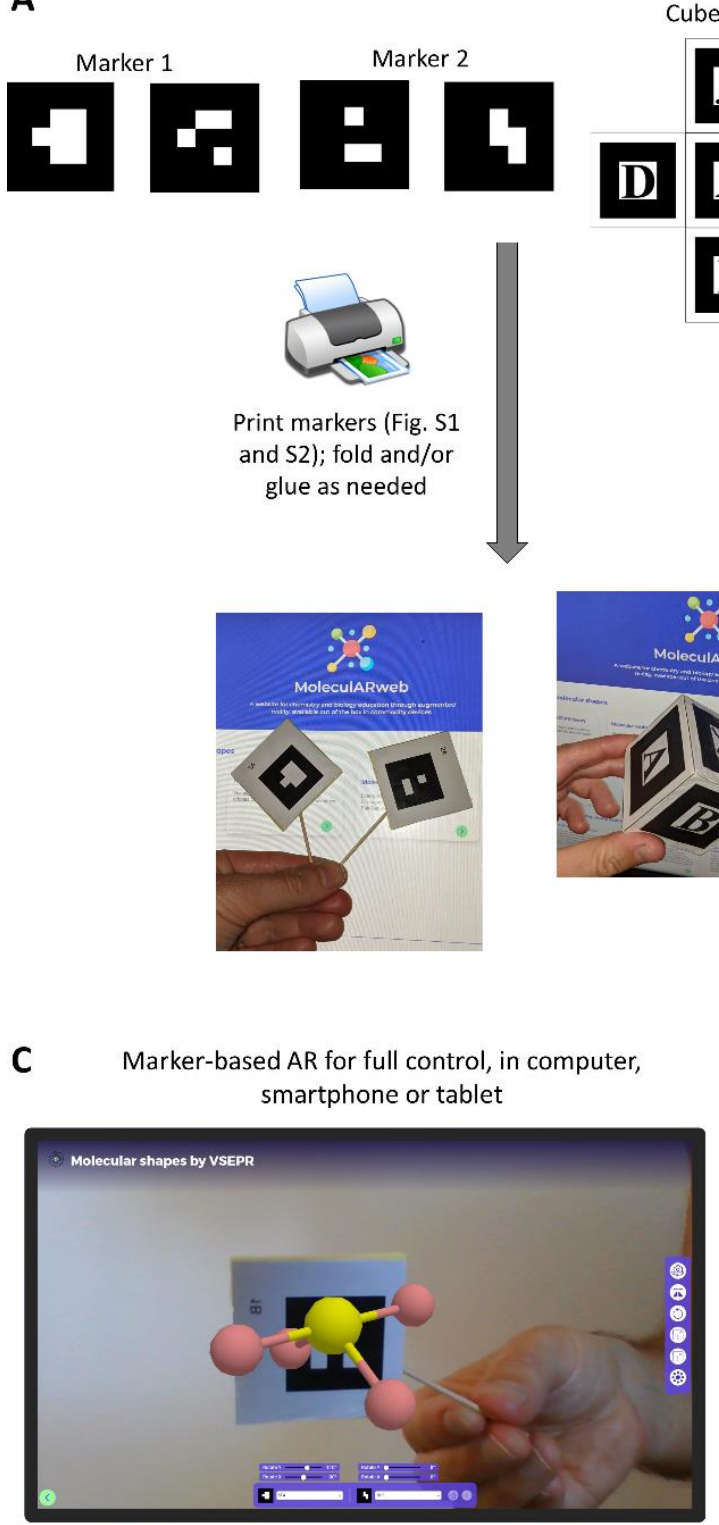

D

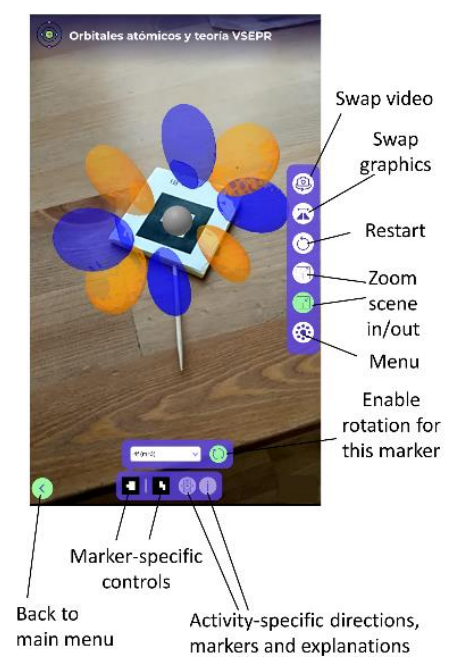

B

\section{스}

A B B

F
Smartphone, tablet, laptop or

desktop computer

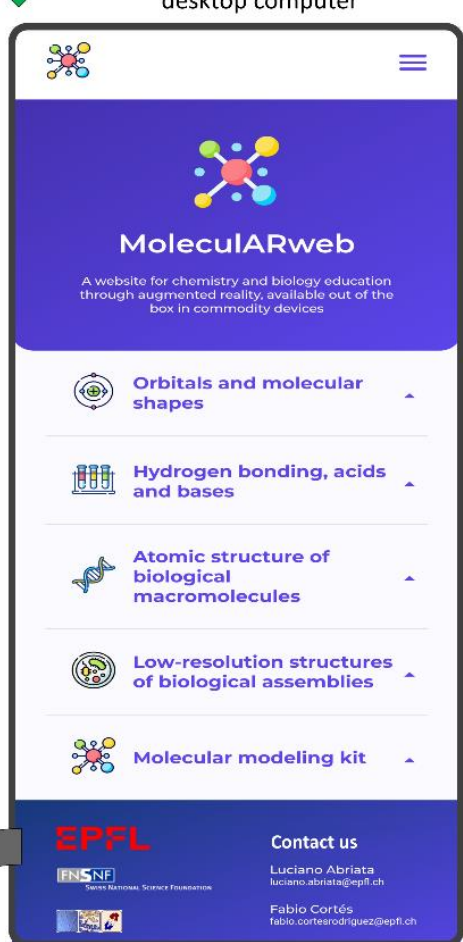

\section{E}

Marker-less AR on smartphone, with objects standing on flat surfaces
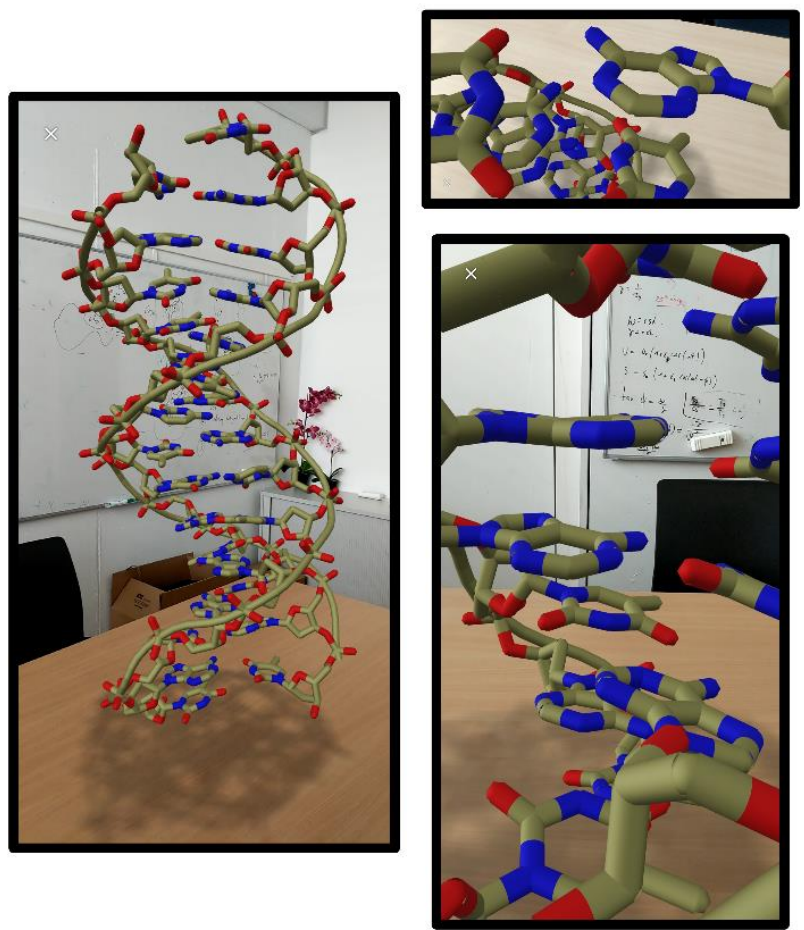

Figure 1. Accessing and using MoleculARweb. (A) Users need to print the markers (from Figure S1 and S2), fold and possibly glue them, and set them in a way physically comfortable for handling. Here, the flat markers 1 and 2 are folded in half and glued onto a flat solid support through which toothpicks cross giving a handle; and the cube marker is folded and glued onto a custom-cut rubber cube. (B) Users then must direct a web browser to https://MoleculARweb.epfl.ch on a smartphone, tablet 
or computer, access an activity from the modules, then enable the webcam when prompted and show it the markers. (C) An $\mathrm{SF}_{4}$ molecule from the activity on molecular shapes based on VSEPR theory, displayed on a marker in a laptop screen. (D) Example of controls in an activity, shown in the smartphone layout in the activity about atomic orbitals here showing a $4 f$ orbital. Controls on the right are general across the different activities and permit controlling general zoom level, activity restart, graphics orientation depending on device (for example using the computer webcam as a mirror or a smartphone for look-through requires opposite video swaps). Controls at the bottom are activity-specific, allowing the selection of different examples and tuning marker-specific rotations and zoom level through touch gestures. (E) For visualization-only activities the web apps can also launch a WebXR mode that does not use AR markers but detects flat surfaces and deposits the models on them, such that the user can explore them all around as shown here for a double-stranded DNA molecule.

\section{Applications active by the time of first release}

As of august 2020 MoleculARweb contains over 20 activities arranged in five main thematic modules: (i) Atomic orbitals, molecular orbitals and molecular shapes; (ii) Hydrogen bonding, acids and bases; (iii) Atomic structure of biological macromolecules; (iv) Large biological assemblies; and (v) A virtualmechanical molecular modeling kit.

The module on atomic orbitals, molecular orbitals and molecular shapes includes five activities. In the first activity users can inspect the $s, p, d, f$ and $g$ atomic orbitals, the $s p, s p^{2}, s p^{3}, s p^{3} d$ and $s p^{3} d^{2}$ hybrid orbitals, and the main ideal VSEPR geometries (Figure $2 \mathrm{~A}$ showing a $3 d z^{2}$ atomic orbital and a trigonal planar molecular geometry). Any orbital or molecular shape can be displayed on any of two markers, even simultaneously, which allows interactive comparison of different objects. The second activity displays all the molecular orbitals precalculated for a list of simple molecules and polyatomic ions: $\mathrm{H}_{2} \mathrm{O}, \mathrm{O}_{2}, \mathrm{BH}_{3}, \mathrm{CH}_{4}, \mathrm{CO}, \mathrm{CO}_{2}, \mathrm{~N}_{2}$, $\mathrm{N}_{3}^{-}, \mathrm{NH}_{3}, \mathrm{NO}_{2}, \mathrm{BF}_{3}, \mathrm{SCN}^{-}$. After selecting a molecule or ion for a marker the user can chose to display one of its orbitals (Figure 2B showing sigma antibonding and pi bonding orbitals of $\mathrm{CO}_{2}$ ). As in the previous example, each marker can independently handle a different molecule, which is here useful to compare orbitals and assist thinking about reactivity. The third activity contains molecular structures representative of the main kinds of molecular shapes according to VSEPR theory, also handled independently on two markers (Figure 2C). The fourth activity is a web remake of the AR examples employed and tested in Martinez-Hung et al, which includes examples of crystalline structures and some key water complexes (Figure 2D). The fifth activity opens up the modeling kit where users can load and work on any molecule, described later as a separate module.

The module on hydrogen bonding, acids and bases includes four activities where teachers can show, or students can explore themselves, how hydrogen atoms interact with lone electron pairs of electronegative atoms through hydrogen bonds, how the proton can actually "jump" between molecules changing their protonation states, and how this relates to concepts of acids and bases through a very graphical, interactive description of the equilibria involved. One example displays two water molecules that can exchange between hydronium, water and hydroxide species by exchanging protons when they are close enough (Figure 3A). Another example shows how acetic acid can protonate water to give hydronium cation, and another how ammonia can take a proton from water producing hydroxide anion (Figure 3B). The fourth example features an acid and a base from two amino acid side chains reacting directly with each other to change from neutral to oppositely charged. These examples highlight the dynamic nature of equilibria, with protons jumping back and forth but overall spending more or less time on each molecule as expected from relative acidic constants.

The module on atomic structure of biological macromolecules includes preset examples of static $3 \mathrm{D}$ visualizations and some interactive activities. The static visualizations are all arranged under the "Example structures" activity, in whose dropdown menus users can chose among a set of examples extended regularly by adding new visualizations rendered with VMD, currently including a short piece of double-stranded DNA, a protein-DNA complex, a short peptide and a drug-bound trimeric protein). These static visualizations can be manipulated either in desktop or laptop computers with the AR markers, or directly in full AR on smartphones, or placed virtually on top of flat surfaces captured from the real world as in Figure 1E displaying a dsDNA molecule that the users can walk around. The interactive activities include an app for inspecting alpha and beta protein secondary structures at atomic resolution while toggling the cartoon representations and the most important interactions that stabilize these structures (Figure 4A); another for docking a DNAbinding protein onto its cognate DNA through its major groove (Figure 4B, exemplifying its use in teaching), and another where students can interactively dock a peptide into an experimental electron density map from an actual X-ray diffraction experiment (Figure S3, introducing how molecular structures are experimentally determined). 

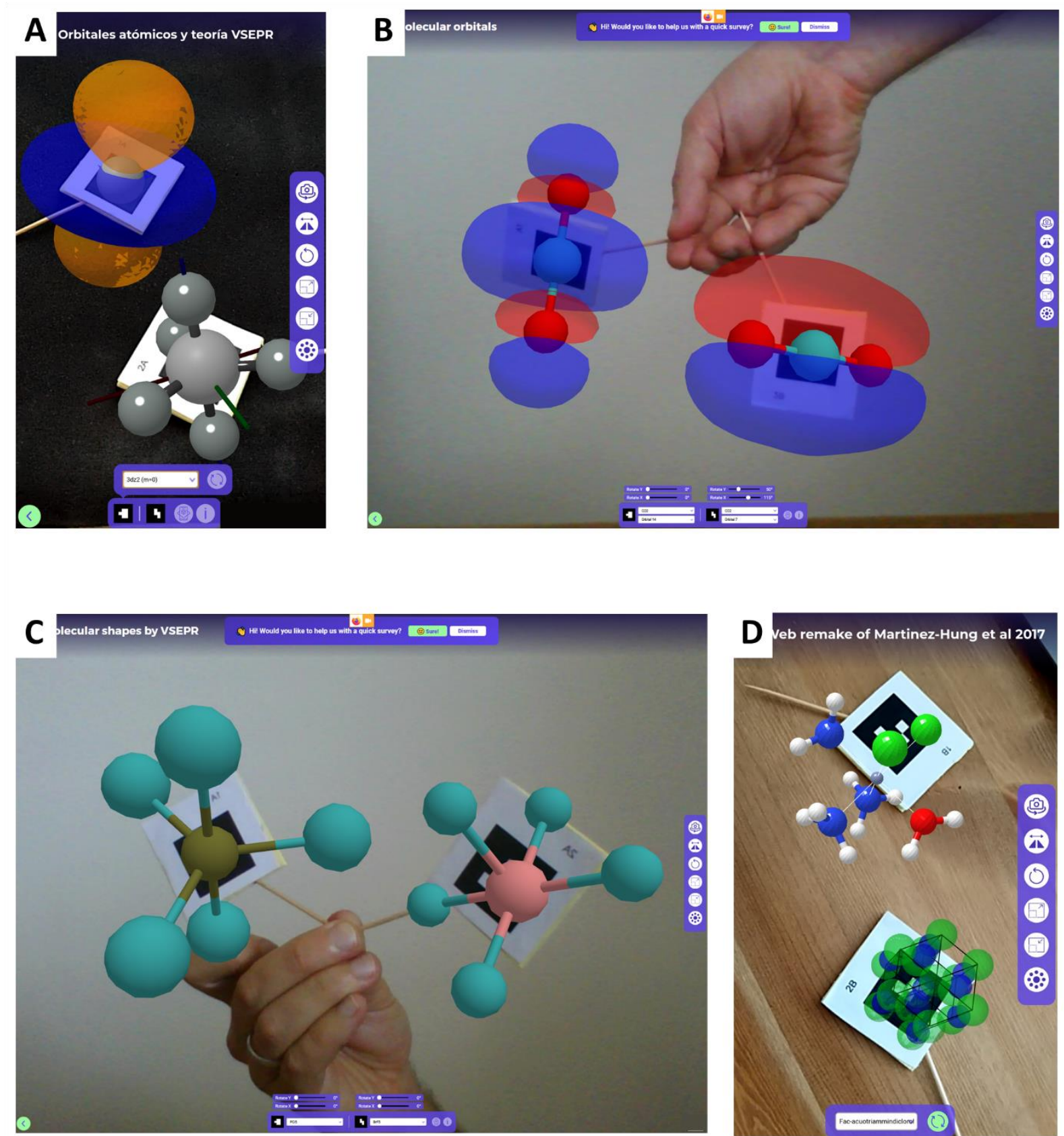

Figure 2. Module on atomic orbitals, molecular orbitals and molecular shapes. (A) Markers displaying a $3 d z^{2}$ atomic orbital and a trigonal bipyramidal geometry, in a smartphone. (B) Markers displaying sigma antibonding (left) and $\pi$ bonding molecular orbitals of $\mathrm{CO}_{2}$ in a computer. (C) Comparing trigonal bipyramidal ( $\left.\mathrm{AX}_{5}\right)$ and square pyramidal geometries $\left(\mathrm{AX}_{5} \mathrm{E}\right)$ of $\mathrm{PCl}_{5}$ and $\mathrm{BrF}_{5}$, respectively, in a computer. (D) Markers displaying the aquodiaminotrichloroferric complex and the unit cell of $\mathrm{ZnS}$ in a smartphone, among other examples from Martinez-Hung et al 2017 repurposed for webAR. 

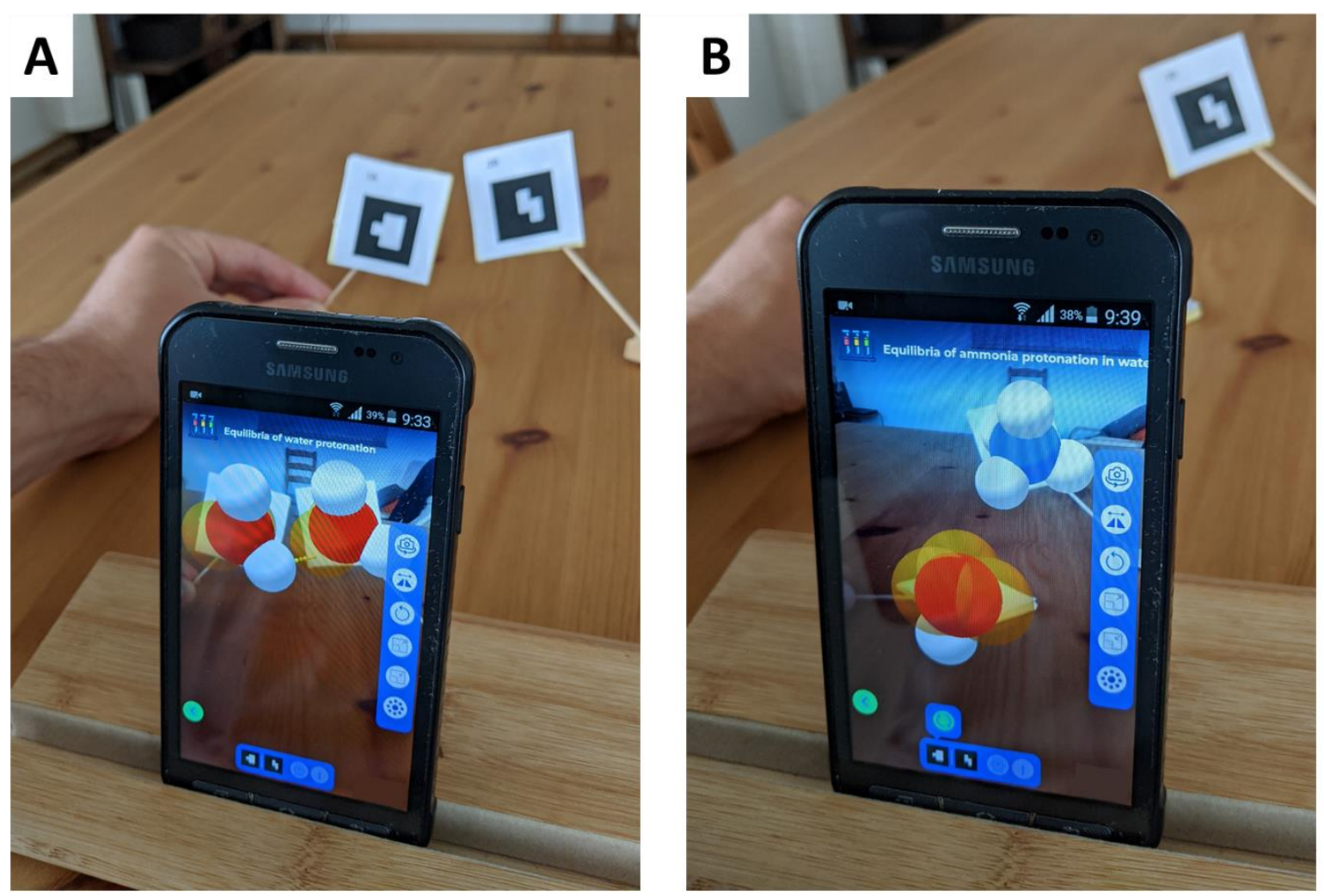

Figure 3. Module on hydrogen bonding, acids and bases. (A) Two water molecules establishing a hydrogen bond and ready to transfer a $\mathrm{H}^{+}$from the left to the right. (B) A water molecule reacted with ammonia leading to a hydroxide anion and an ammonium cation. The activities of this module utilize CPK color scheme for atoms: red for oxygen, blue for nitrogen, grey for carbon and white for hydrogens; while lone electron clouds are shown in transparent orange. This figure further illustrates a convenient use of smartphones and tablets where they are left standing out on a support freeing up both hands for molecule handling.

A separate module contains static visualizationonly material about biological macromolecular assemblies, to be viewed with AR markers in any device or standing on flat surfaces detected by the app without any markers in smartphones. At the moment of publication this module includes a 3D volumetric map of a bacteriophage that has punched its needle through the two bacterial membranes (EMD-9010, Figure 5A, several marker-less views of the same standing object, with annotations); an integrative model of the SARS-Cov-2 spike protein bound to human ACE2 (PDB IDs 6VYB, 6M17 and 6M0J); a structure of a bacterial pilus determined by cryoelectron tomography with X-ray structures fitted inside and including portions of the two cell membranes and the peptidoglycan (PDB 3JC8 + EMD-3247, Figure 5B, held on a marker and seen on a computer); an X-ray structure of a nucleosome including the 4 histones and 2 rounds of dsDNA wrapped around (PDB ID 1AOI, Figure 5C, held with marker in a smartphone); a cryo-electron tomography map of the human nuclear pore complex obtained in situ (EMDB-3103, Figure 5D, on marker in smartphone); and a cryo-electron microscopy structure of a mammal ribosome with a tRNA in the $\mathrm{A} / \mathrm{P}$ position paired to a stretch of mRNA and another tRNA in the P/E position (PDB 6HCJ, Figure 5E). 
A
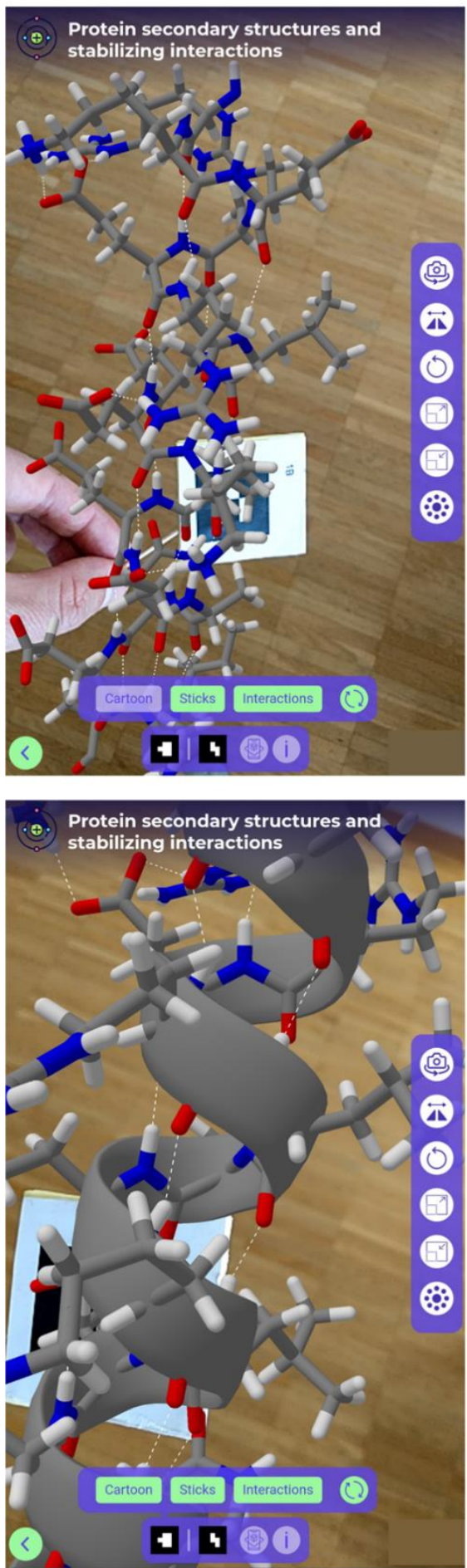

B
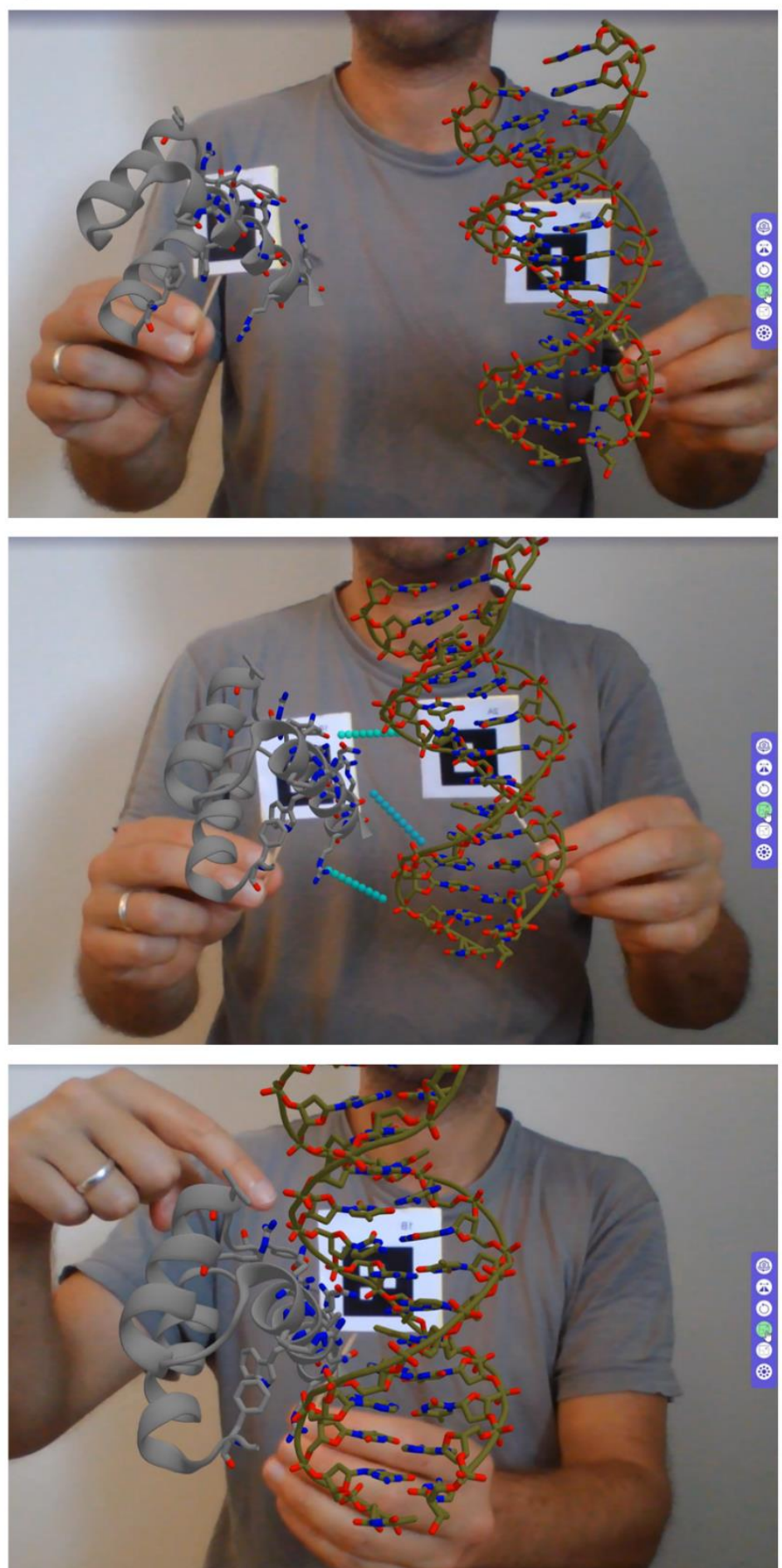

Figure 4. Module on atomistic structure of biological macromolecules. (A) Inspecting a fully atomistic $\alpha$ helical structure on a smartphone, toggling the depiction of interactions (compare left and right). (B) Teaching about DNA structure and proteinDNA interactions during a remote online class on a computer: while both molecules are away (or only one marker is shown to the webcam) each can be inspected individually in detail; then when they approach the program highlights interactions which when satisfied lead to the protein-DNA complex. In the complex, the instructor here points with his fingers at the most important interactions, here salt bridges between positively charged side chains of the protein and the negatively backbone phosphates of the double-stranded DNA molecule. This example, from PDB 1FJL, intends to aid understanding of noncovalent interactions, here especially guided by electrostatic complementarity between positively charged amino acids of the protein and phosphate groups of the DNA molecule. 

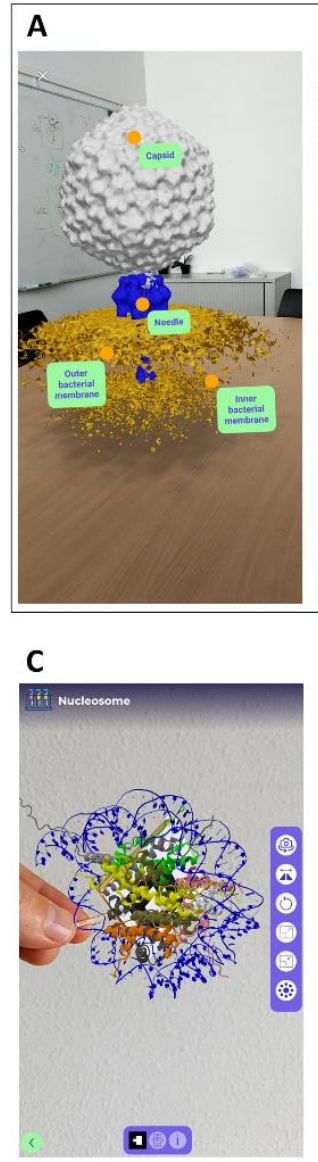

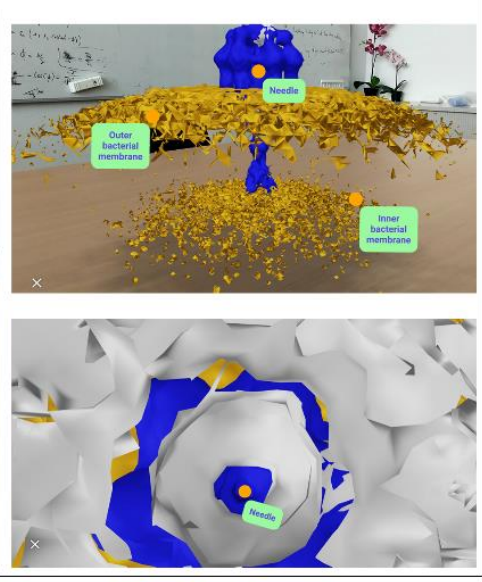

D

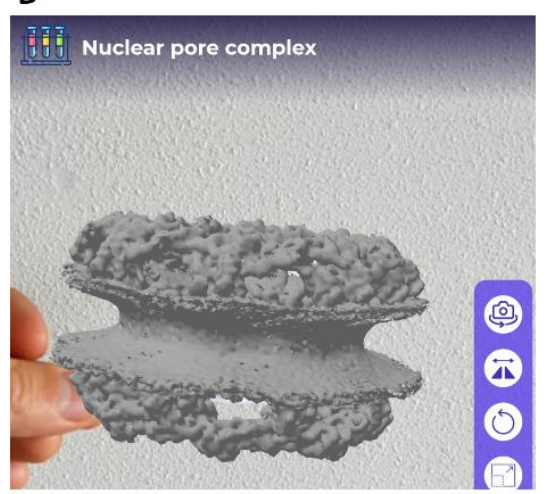

B

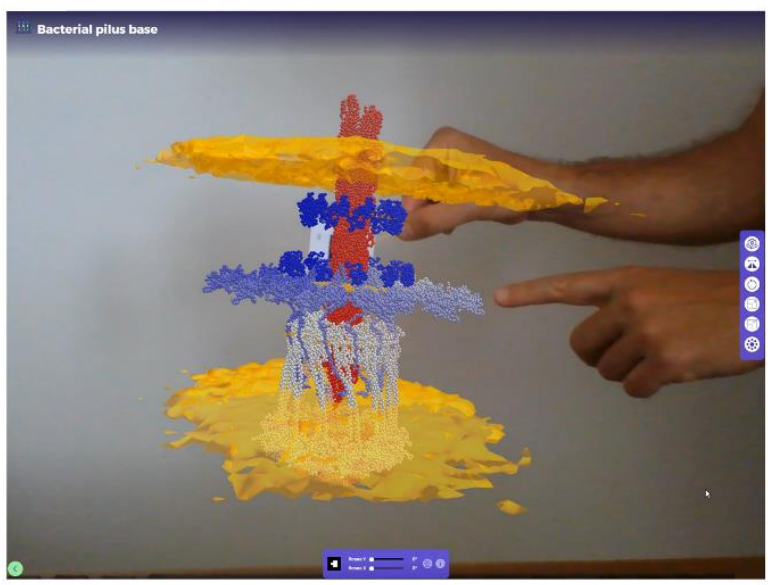

E

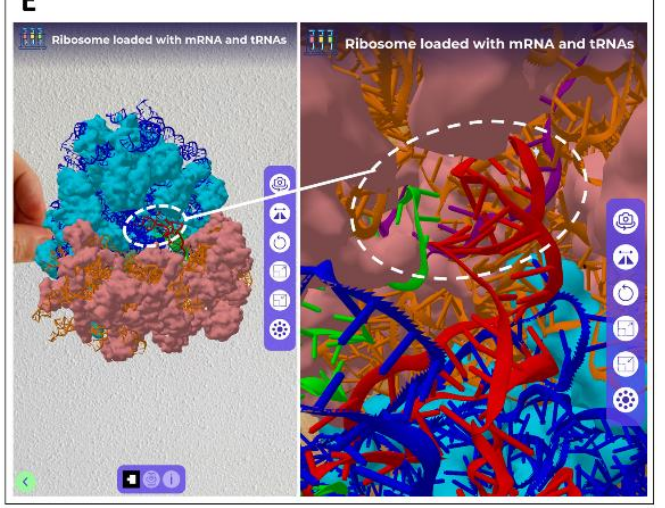

Figure 5. Module on biological macromolecular assemblies. (A) A bacteriophage with its needle punching through the outer and inner membranes of a bacterium as observed by 3D cryo-electron tomography (EMDB-9010), here displayed on markerless AR on a flat surface with overlaid labels that name the different parts of the virus. Three different views of the same session are shown, two from outside the virus and one from inside the capsid looking down towards the needle. (B) Displayed on a computer, a cryo-electron tomogram of the bacterial pilus machine obtained in intact cells with X-ray structures of proteins fit inside and including portions of the two cell membranes (orange surfaces) and peptidoglycan (pointed at by the finger) (example built from PDB 3JC8 and EMD-3247). (C) X-ray structure of a nucleosome including the 4 histones and 2 rounds of double-strand DNA wrapped around (PDB ID 1AOI) held with an AR marker in a smartphone. (D) Cryo-electron tomography of the human nuclear pore complex obtained in situ (EMDB-3103, Figure 5D, on marker in smartphone. (E) A mammal ribosome held on a marker (PDB 6HCJ) seen from far (left) with the large subunit in cyan-blue shades and the small subunits in orange-salmon shades, proteins as surfaces and RNA molecules as cartoons; and zoomed into the tRNA-mRNA interaction sites (right) where the mRNA is in purple, the tRNA at the A/P position is in green, and the tRNA at the P/E position is in red.

The last module is not a specific activity but rather a web page where users can view and handle in AR any molecule from its atomic coordinates, as a virtual alternative to a tangible molecular modeling kit (Figure 6A). The molecules are input in PDB format (a very handy web tool to retrieve or even build any molecule is Prof. Hanson's web app at https://chemapps.stolaf.edu/jmol/jsmol/hackamol.ht

$\underline{m})$. Several molecules are preset as examples in the AR web app, ranging from very small molecules useful for an introduction to molecular shapes and structure, to small-molecule pharmaceuticals and metabolites, pieces of dsDNA, proteins, nanoparticles and carbon allotropes, among others. The app displays atoms as either spheres or sticks (Figure 6B), with standard CPK colors. Double bonds are displayed as thick sticks. Beyond mere visualization, the app simulates thermal fluctuations, which by coupling throughout multiple atoms give rise to torsions and thus small conformational transitions. The observed motions are propagated from a very simple rigid-body mechanical force field after connecting atoms with a special set of constraints as described in Methods. Of course, such simple approach cannot accurately describe the full underlying physics, especially non-classical phenomena, being closer to a tangible, physical molecular modeling kit. Some advantages we expect from this app over a physical kit are the virtually unlimited number and types of atoms available, the possibility to immediately explore complex molecules without the need to build them, and the 
communication of the idea that molecules are dynamic rather than static, directly allowing for visualization of isomers, dihedral rotations, the rigidity of double bonds and rings, and other concepts about molecular conformations. Example use cases of this app are inspecting dihedral angles as molecules move, useful to understand for example cis-/transisomerization and Newman projections in butane (Figure 6B) or chair-boat transitions in 6-member rings like cyclohexane (Figure $6 \mathrm{C}$ ); also looking at complex molecular geometries and chirality as exemplified for cholesterol (Figure 6D), or the atomic details of any biological molecule or material as in the preset examples shown in Figure 7: proteins, nucleic acids, carbon nanotubes, and crystalline arrangements.

\section{User adoption}

The use of AR technology in chemistry curricula has already shown pedagogical success in multiple reports, ${ }^{12,31-34}$ even through as far as we know they all involve only static content i.e. not featuring any molecular motions, interactions or any kind of interactivity as our website does, they do not stand web-accessible, most are rather limited in the number of available examples and activities, and a few require ad hoc peripherals. We expect that our website, with several activities spanning many concepts and going beyond mere static visualization, will be of great use for students, educators and science communication. So far, a prototype version of the website available since May 2019 only in English until release of the formal website has been accessed >6,000 times as of September 2020, from all over the world as shown in Figure S4 (the old website now redirects to the new one). Among these users there are several high school and early university teachers who contacted us thanking for the availability of the tools, asking questions and proposing several ideas now implemented, and, very relevant, attesting good engagement from students. The free, open availability on the web was especially welcome, as observed in other widely accessed webbased science education resources. ${ }^{35-37}$

Among the activities implemented in MoleculARweb those about molecular shapes and coordination chemistry from Martinez-Huang et al. have proved their pedagogical value in a previous non-web implementation. ${ }^{20} \mathrm{We}$ further tested user engagement of some of the other activities on students and the general public during EPFL's public outreach and science communication ("open doors") event in 2019

(https://www.epfl.ch/campus/events/celebrationen/portes-ouvertes/), during EPFL's visit to high schools (https://memento.epfl.ch/event/journeethematique-au-lycee-cantonal-de-porrentruy/) and to ETHZurich during 2019, and in the 2019 course on Structural Inorganic Chemistry at the affiliation of GF and DDM. We observed in all cases strong engagement and enthusiasm, and very quick adaptation to the use of the markers especially by kids, teenagers and young adults. Notably, some teenagers were particularly interested in the programming aspects of the website, having realized that they could seamlessly view and experiment with the source code on their computers.

In the science communication event for the general public, each visitor to our stand toured individually through four AR activities depicted as "stations" in Figure 8 . Each activity covered increasingly complex subjects starting from electrostatic interactions between small molecules, then the role of these interactions in biology specifically in protein-DNA binding, then moving on to large assemblies like viruses and finally to a quick game about how protein structures are determined experimentally (where they docked amino acids to an electron density cloud building up a short peptide). The last activity served as an introduction to a hands-on experiment at the next-door stand, where visitors crystallized lysozyme, looked at the crystals and then were instructed about how electron densities are determined by X-ray diffraction. 

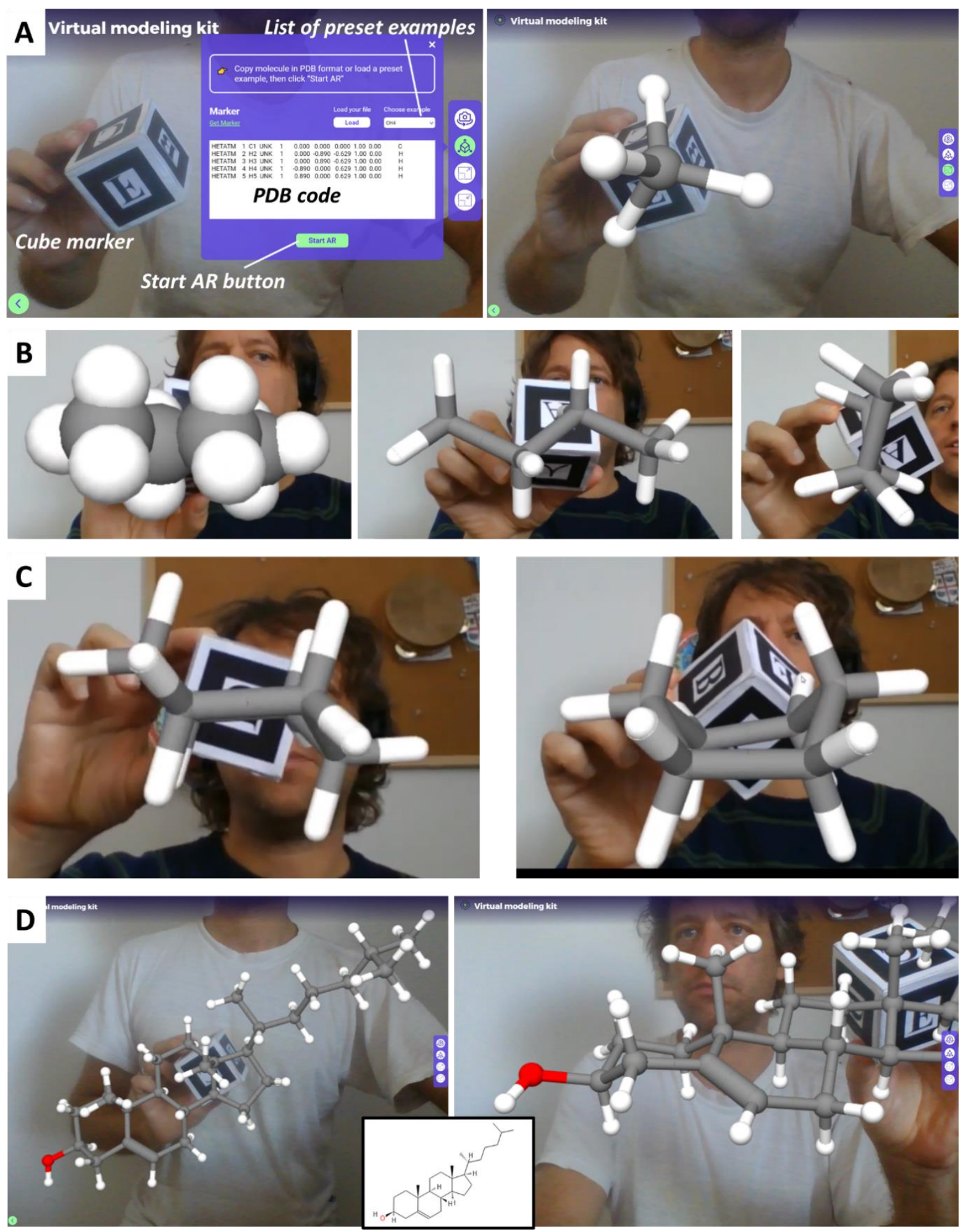

Figure 6. Virtual Molecular Modeling Kit. In this web app users can load any molecule and then handle them in 3D with a cube marker; furthermore, the web app incorporates rudimentary molecular mechanics that make models flexible allowing users to study motions. (A) The main interface showing controls to load a molecule in PDB format and start the AR experience, before (left) and after (right) clicking on "Start AR" to display a methane molecule. (B) The starting butane molecule from the preset example in its lowest-energy anti conformation, shown in spacefill representation (left) and as sticks (center); and in the syn conformation observed after the molecule has moved for some time due to thermal fluctuations. (C) Cyclohexane in its chair conformation as in the starting coordinates from the preset example, and then in a boat conformation obtained as the molecule moves due to thermal motions. (D) A molecule of cholesterol (from the NCI database, accessed through Prof. Hanson's Hack-A-Mol: https://chemapps.stolaf.edu/jmol/jsmol/hackamol.htm) shown in full on the left, and zoomed on the $\mathrm{OH}$ side as the instructor comments on the only double bond (thicker bond in front) and the chirality of the $\mathrm{OH}-$ bound and methyl-bound carbons. 

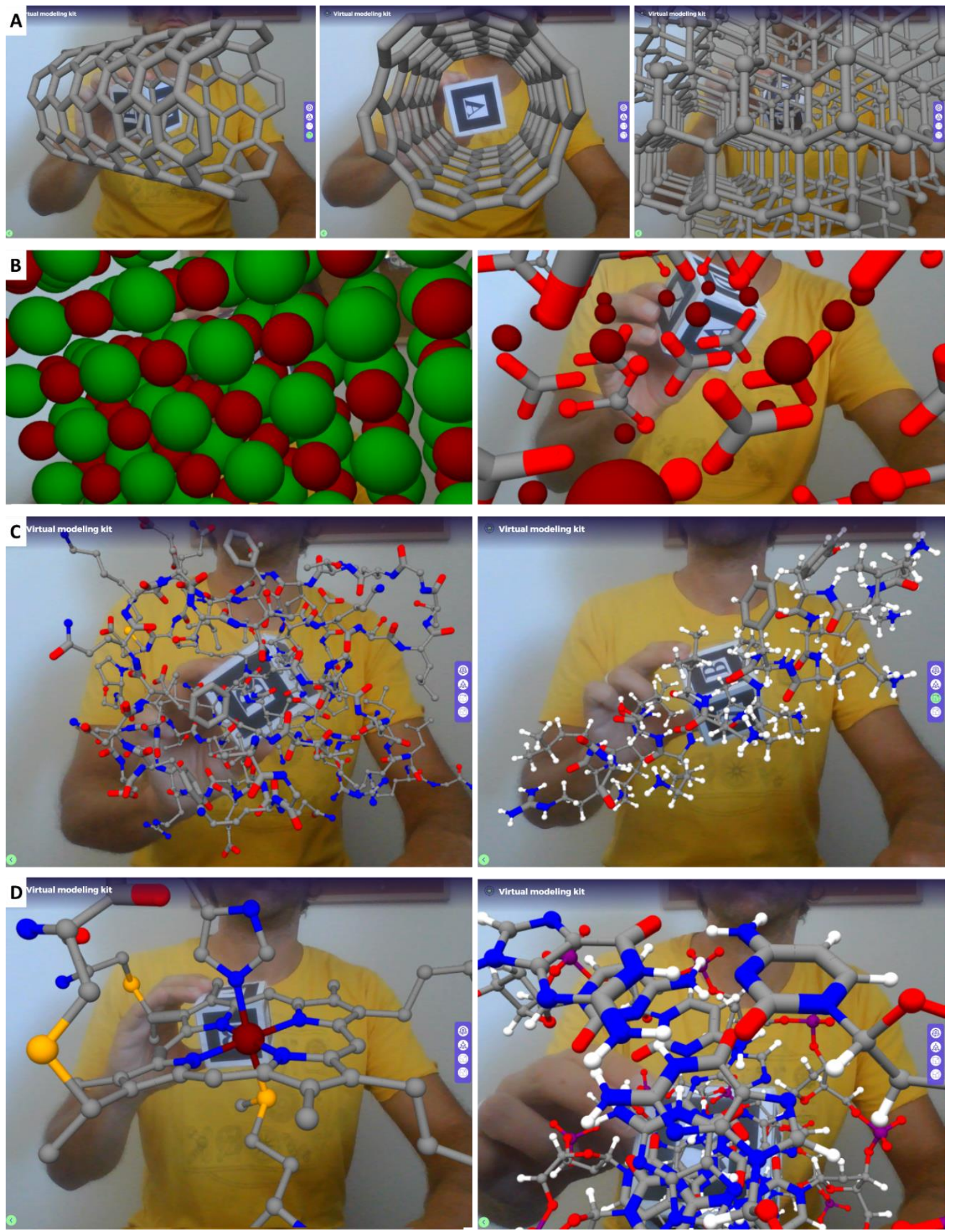

Figure 7. Virtual Molecular Modeling Kit used on larger molecules. (A) Two views of a carbon nanotube (left and center, built with CHARMM-GUI); and a diamond crystal (right) built from a PDB file retrieved at https://preparatorychemistry.com/Jmol_carbon_diamond.htm. (B) Crystal arrangements in sodium chloride (left, sodium and chloride ions shown as red and green spheres, respectively) and calcite $(\mathrm{CaCO} 3$, right, with calcium cations as spheres and carbonate anions as sticks) built from PDB files available at https://ww2.chemistry.gatech.edu/ 1w26/structure/small_molecules/index.html. (C) Ubiquitin as in the X-ray structure in PDB 2D3G (left) and an alpha helical antimicrobial peptide solved by NMR in PDB 2RLH (right). Notice the double-bond character of peptide bonds and aromatic rings, also the hydrogen bonding patterns in the helix. (D) NMR structure of a doublestrand DNA (PDB 5J3G) seen from far (left) to inspect overall shape, major and minor grooves, and inspected closely for base stacking and hydrogen bonding patterns (right). 
The Structural Inorganic Chemistry course taught by DDM employed mainly the activity about molecular orbitals, which helped students to navigate the molecular orbitals of several small molecules studied in class and inspect these orbitals in 3D. This course, at the fourth year of the Diploma in Chemistry, seeks to develop the knowledge needed to structurally characterize inorganic compounds, providing the tools for the interpretation of their topology, reactivity and molecular electronic structure. Students get familiarized with basic concepts of symmetry and group theory and are trained to understand different models used to describe the molecular electronic structure and chemical-physical properties such as reactivity and molecular spectroscopy of inorganic compounds. In particular, the model of linear combination of atomic orbitals is developed in detail to obtain delocalized molecular orbitals. The molecular orbitals obtained for a set of typical inorganic topologies, displayed in AR on our website, are used in the interpretation of the chemistry of inorganic compounds from a structural / thermodynamic point of view, as students explain stability and reactivity by recognize intermolecular interactions allowed by symmetry and reaction sites. Thus, by comparing pairs of molecules and orbitals held on the AR markers, students could decide on the plausible reactions and geometries, acting as an extension of the standard on-paper exercises.

We are now collaborating with high-school and early university teachers who are employing the website for their courses. Our usage data shows that the most accessed activity is the molecular modeling kit, where teachers show their students many of the molecules that are now available as preset examples and also instruct them to build and inspect their owns.

For wider assessment of the utility of the website, we have implemented ways to monitor activity and utility as perceived by students and by educators in the website itself. Namely, the official website automatically collects usage statistics to help us improve user coverage in future updates (mainly device type, operating system and web browser), and regularly prompts optional surveys specifically designed for students and for educators, whose results will help us evaluate the functionality and utility of the activities, user experience, and active effect on learning.
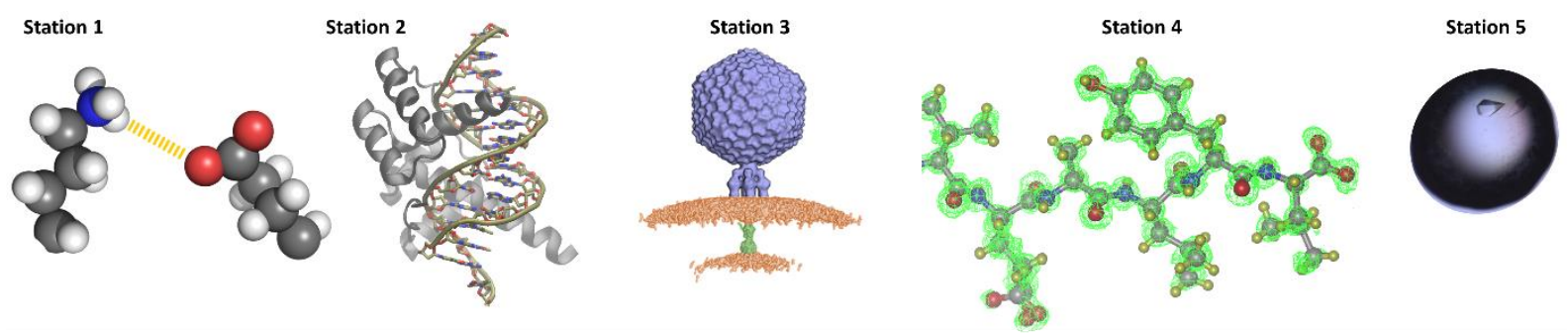

Figure 8. Augmented reality activities implemented in a public outreach day at EPFL. Members of the public individually tried augmented reality apps as they were first instructed about electrostatic interactions between charged molecules (station 1), then about their role in essential biological processes like reading DNA information by proteins (station 2), and then about how proteins and DNA make up larger assemblies such as virus which in turn can interact with membranes in this case to inject DNA into the host (station 3). The activity in station 4 addressed the problem of how structures are determined experimentally, before moving to station 5 at the nextdoor stand of the Protein Production and Structure Core Facility where the public could grow their own lysozyme crystals and watch them under a microscope.

\section{Closing remarks for educators}

We here introduced MoleculARweb with a large set of interactive pedagogical activities for chemistry and structural biology education in a fully web interface, accessible for free without registration and in six languages. We propose multiple ways to use the website, most illustrated in the examples above: (i) teaching students and the public in live presentations assisted by large TV screens or beamers and (ii) in online teaching and in science communication videos by capturing the browser displays, in both cases the educator handling the molecules; (iii) instructing students themselves to explore the models with handheld markers or even by employing markers inserted in the texts provided to them; (iv) and by setting visualizations on flat surfaces like the floor, table or workbench, either shared through large markers lying on the surface or individually. We note that marker- 
based activities are suitable for finely handling objects with the hands and to trigger interactivity between pairs of objects in the interactive examples; besides, multiple independent users using the same AR marker seen through different devices will display the virtual objects from their respective locations, so they can be applied to collaborative work. On the other hand, models standing on flat surfaces are not locked to any marker so the views cannot be shared by independent users, but they are more practical to look at very large models, as one can easily move around them to inspect them from any angle and position, even getting inside of them as in the bacteriophage example in Figure 5A.

We strongly encourage educators to test the website, implement it in their lessons both for direct teaching in person or through virtual courses, and also by setting students to work by themselves, stressing that the server is available everywhere so students can use it at their homes too. We also encourage all users to answer the optional surveys that pop up occasionally (with distinct questions for students and educators), as they will allow us to improve the website and maintain it functional for the devices and browsers most widely used. Moreover, we expect that the information from the surveys will allow us to carry out a large-scale assessment about the utility and effects of the website, and more broadly of webAR technologies, on chemistry education. We further encourage educators to contact us to point out bugs, ask for questions and troubleshooting, and to propose new activities.

\section{Methods}

The server is based entirely in client-side programming i.e. HTML5, CSS and JavaScript code. As such it can be easily copied for reuse from alternative locations and even adapted to run locally instead of online. to https://MoleculARweb.epfl.ch. All code and 3D objects can be consulted and downloaded directly from the website and at GitHub: https://github.com/fcor/molecular-mirror-app. Key to the development of MoleculARweb are A-Frame, AR.js, Three.js, jsartoolkit and Cannon.js among other libraries, as described in ${ }^{26}$. Visualizations standing on flat surfaces are achieved through the Model-Viewer web component. Interactivity is handled through ad hoc JavaScript code that can be consulted directly from the source code of the web pages. Molecular motions in the virtual molecular modeling kit are handled by Cannon.js; more specifically, atoms are represented by hard spheres of radii proportional to atomic radii, pairs of atoms within bonding distance are kept at a fixed distance with a strong Cannon constraint, pairs of atoms bonded to the same atom are weakly constrained to the starting distances to maintain angles, and pairs of atoms bonded to pairs of bonded $\mathrm{sp}^{2}$ carbons are also constrained to hamper rotations around double $\mathrm{C}-\mathrm{C}$ bonds. We do not provide further technical details here as we intend to focus on the actual activities available in the website as of August 2020.

The 3D objects representing small molecules are built on-the-fly using 3D graphics primitives, while complex 3D objects were exported from VMD (structures, electron maps, molecular orbitals, etc.) or Orbital Viewer version 1.04 (atomic orbitals) and edited as required with Blender. To compute molecular orbitals, the small molecules were built with Molden $^{38}$ and then optimized and molecular orbitals calculated at HF/STO-3G level with the Gaussian 09 package. Optimized structures and molecular orbitals were rendered in VMD. ${ }^{39}$

\section{Acknowledgements}

This work is funded by EPFL and by Spark grant CRSK-1_190376 to LAA in a project hosted by EPFL's Laboratory for Biomolecular Modeling led by MDP. The molecular orbital calculations were carried out using the resources of the Computer Center of CCT-Rosario, member of the National System of High-Performance Computing (SNCAD, MincyT-Argentina). We thank the developer and user communities behind AR.js, jsartoolkit, A-Frame, I18Next, three.js, Cannon.js, Model-Viewer, especially N. Carpignoli, J. Ethienne, D. McCurdy and S. Hedman. We also thank D. Vanegas for help with user interface design, and A. Prunotto and S. Mayer for help with translations into Italian and German.

The icons used in the website were downloaded from Iconscout.com and Flaticon.com, made by Pixel Perfect, Smashicons, Freebies, Diacanvas Studio and Dario Ferrando (all available under the most open, free license of each provider). The website's logo was composed from free, open icons taken from the same authors at Flaticon.com.

\section{References}

(1) White, B.; Kim, S.; Sherman, K.; Weber, N. Evaluation of Molecular Visualization Software for Teaching Protein Structure Differing Outcomes from Lecture and Lab: Differing Outcomes 
from Lecture and Lab. Biochem. Mol. Biol. Educ. 2002, 30 (2), 130-136.

https://doi.org/10.1002/bmb.2002.494030020026.

(2) Jaswal, S. S.; O’Hara, P. B.; Williamson, P. L.; Springer, A. L. Teaching Structure: Student Use of Software Tools for Understanding Macromolecular Structure in an Undergraduate Biochemistry Course. Biochem. Mol. Biol. Educ. 2013, 41 (5), 351-359.

https://doi.org/10.1002/bmb.20718.

(3) Terrell, C. R.; Listenberger, L. L. Using Molecular Visualization to Explore Protein Structure and Function and Enhance Student Facility with Computational Tools. Biochem. Mol. Biol. Educ. 2017, 45 (4), 318-328. https://doi.org/10.1002/bmb.21040.

(4) Niece, B. K. Custom-Printed 3D Models for Teaching Molecular Symmetry. J. Chem. Educ. 2019, 96 (9), 2059-2062. https://doi.org/10.1021/acs.jchemed.9b00053.

(5) de Cataldo, R.; Griffith, K. M.; Fogarty, K. H. Hands-On Hybridization: 3D-Printed Models of Hybrid Orbitals. J. Chem. Educ. 2018, 95 (9), 1601-1606.

https://doi.org/10.1021/acs.jchemed.8b00078.

(6) Copolo, C. E.; Hounshell, P. B. Using Three-Dimensional Models to Teach Molecular Structures in High School Chemistry. J. Sci. Educ. Technol. 1995, 4 (4), 295-305.

https://doi.org/10.1007/BF02211261.

(7) Shelton, B. E. How Augmented Reality Helps Students Learn Dynamic Spatial Relationships; University of Washington Seattle, 2003.

(8) O’Connor, M.; Deeks, H. M.; Dawn, E.; Metatla, O.; Roudaut, A.; Sutton, M.; Thomas, L. M.; Glowacki, B. R.; Sage, R.; Tew, P.; Wonnacott, M.; Bates, P.; Mulholland, A. J.; Glowacki, D. R. Sampling Molecular Conformations and Dynamics in a Multiuser Virtual Reality Framework. Sci. Adv. 2018, 4 (6), eaat2731. https://doi.org/10.1126/sciadv.aat2731.

(9) M. Krichenbauer; G. Yamamoto; T. Taketom; C. Sandor; H. Kato. Augmented Reality versus Virtual Reality for 3D Object Manipulation. IEEE Trans. Vis. Comput. Graph. 2018, 24 (2), 1038-1048. https://doi.org/10.1109/TVCG.2017.2658570.

(10) Gillet, A.; Sanner, M.; Stoffler, D.; Goodsell, D.; Olson, A. Augmented Reality with Tangible Auto-Fabricated Models for Molecular Biology Applications. IEEE Visualization 2004.

(11) Gillet, A.; Sanner, M.; Stoffler, D.; Olson, A. Tangible Interfaces for Structural Molecular Biology. Struct. Lond. Engl. 1993 2005, 13 (3), 483-491.

https://doi.org/10.1016/j.str.2005.01.009.

(12) Berry, C.; Board, J. A Protein in the Palm of Your Hand through Augmented Reality. Biochem. Mol. Biol. Educ. Bimon. Publ. Int. Union Biochem. Mol. Biol. 2014, 42 (5), 446-449. https://doi.org/10.1002/bmb.20805.

(13) Vega Garzón, J. C.; Magrini, M. L.; Galembeck, E. Using Augmented Reality to Teach and Learn Biochemistry. Biochem. Mol. Biol. Educ. Bimon. Publ. Int. Union Biochem. Mol. Biol. 2017. https://doi.org/10.1002/bmb.21063.

(14) Wolle, P.; Müller, M. P.; Rauh, D. Augmented Reality in Scientific Publications-Taking the Visualization of 3D Structures to the Next Level. ACS Chem. Biol. 2018, 13 (3), 496-499. https://doi.org/10.1021/acschembio.8b00153.

(15) Hirst, J. D.; Glowacki, D. R.; Baaden, M. Molecular Simulations and Visualization: Introduction and Overview. Faraday Discuss 2014, 169 (0), 9-22. https://doi.org/10.1039/C4FD90024C.

(16) Maier, P.; Tönnis, M.; GudrunKlinker, D. Dynamics in Tangible Chemical Reactions. In World Academy of Science, Engineering and Technology; 2009; Vol. 57.

(17) Maier, P.; Klinker, G. Augmented Chemical Reactions: 3D Interaction Methods for Chemistry. Int. J. Online Eng. 2013, 9.

(18) Ratamero, E. M.; Bellini, D.; Dowson, C. G.; Römer, R. A. Touching Proteins with Virtual Bare Hands : Visualizing Protein-Drug Complexes and Their Dynamics in Self-Made Virtual Reality Using Gaming Hardware. J. Comput. Aided Mol. Des. 2018, 32 (6), 703-709. https://doi.org/10.1007/s10822-018-0123-0.

(19) Müller, C.; Krone, M.; Huber, M.; Biener, V.; Herr, D.; Koch, S.; Reina, G.; Weiskopf, D.; Ertl, T. Interactive Molecular Graphics for Augmented Reality Using HoloLens. J. Integr. Bioinforma. 2018, 15 (2). https://doi.org/10.1515/jib-2018-0005. 
(20) Martínez-Hung, H.; García-López, C. A.; Escalona-Arranz, J. C. Augmented Reality Models Applied to the Chemistry Education on the University (Article in Spanish). Rev. Cuba. Quím. 2016, 29 (1), 13-25.

(21) Balo, A. R.; Wang, M.; Ernst, O. P. Accessible Virtual Reality of Biomolecular Structural Models Using the Autodesk Molecule Viewer. Nat. Methods 2017, 14 (12), 1122-1123. https://doi.org/10.1038/nmeth.4506.

(22) Goddard, T. D.; Brilliant, A. A.; Skillman, T. L.; Vergenz, S.; Tyrwhitt-Drake, J.; Meng, E. C.; Ferrin, T. E. Molecular Visualization on the Holodeck. J. Mol. Biol. 2018. https://doi.org/10.1016/j.jmb.2018.06.040.

(23) O'Connor, M.; Deeks, H. M.; Dawn, E.; Metatla, O.; Roudaut, A.; Sutton, M.; Thomas, L. M.; Glowacki, B. R.; Sage, R.; Tew, P.; Wonnacott, M.; Bates, P.; Mulholland, A. J.; Glowacki, D. R. Sampling Molecular Conformations and Dynamics in a Multiuser Virtual Reality Framework. Sci. Adv. 2018, 4 (6), eaat2731. https://doi.org/10.1126/sciadv.aat2731.

(24) Goddard, T. D.; Huang, C. C.; Meng, E. C.; Pettersen, E. F.; Couch, G. S.; Morris, J. H.; Ferrin, T. E. UCSF ChimeraX: Meeting Modern Challenges in Visualization and Analysis. Protein Sci. Publ. Protein Soc. 2018, 27 (1), 14-25. https://doi.org/10.1002/pro.3235.

(25) Stone, J. VMD Support for VR and Interactive MD; https://www.ks.uiuc.edu/Research/vmd/allversions/interactive_MD.html; https://www.ks.uiuc.edu/Research/vmd/allversions/interactive_MD.html: https://www.ks.uiuc.edu/Research/vmd/allversions/interactive_MD.html, 2019.

(26) Abriata, L. A. Building Blocks for Commodity Augmented Reality-Based Molecular Visualization and Modeling in Web Browsers. PeerJ Comput. Sci. 2020, 6, e260.

(27) B. Bach; R. Sicat; J. Beyer; M. Cordeil; H. Pfister. The Hologram in My Hand: How Effective Is Interactive Exploration of 3D Visualizations in Immersive Tangible Augmented Reality? IEEE Trans. Vis. Comput. Graph. 2018, 24 (1), 457-467. https://doi.org/10.1109/TVCG.2017.2745941.

(28) Goddard, T. D.; Brilliant, A. A.; Skillman, T. L.; Vergenz, S.; Tyrwhitt-Drake, J.; Meng, E. C.; Ferrin, T. E. Molecular Visualization on the Holodeck. J. Mol. Biol. 2018, 430 (21), 3982 3996. https://doi.org/10.1016/j.jmb.2018.06.040.

(29) Abriata, L. A.; Rodrigues, J. P. G. L. M.; Salathé, M.; Patiny, L. Augmenting Research, Education, and Outreach with Client-Side Web Programming. Trends Biotechnol. 2018, 36 (5), 473-476. https://doi.org/10.1016/j.tibtech.2017.11.009.

(30) Abriata, L. A. Web Apps Come of Age for Molecular Sciences. Informatics 2017, 4 (3), 28.

(31) Plunkett, K. N. A Simple and Practical Method for Incorporating Augmented Reality into the Classroom and Laboratory. J. Chem. Educ. 2019, 96 (11), 2628-2631. https://doi.org/10.1021/acs.jchemed.9b00607.

(32) Sanii, B. Creating Augmented Reality USDZ Files to Visualize 3D Objects on Student Phones in the Classroom; ACS Publications, 2019.

(33) Eriksen, K.; Nielsen, B. E.; Pittelkow, M. Visualizing 3D Molecular Structures Using an Augmented Reality App. J. Chem. Educ. 2020, 97 (5), 1487-1490. https://doi.org/10.1021/acs.jchemed.9b01033.

(34) Peterson, C. N.; Tavana, S. Z.; Akinleye, O. P.; Johnson, W. H.; Berkmen, M. B. An Idea to Explore: Use of Augmented Reality for Teaching Three-Dimensional Biomolecular Structures. Biochem. Mol. Biol. Educ. Bimon. Publ. Int. Union Biochem. Mol. Biol. 2020, 48 (3), 276-282. https://doi.org/10.1002/bmb.21341.

(35) Camel, V.; Maillard, M.-N.; Piard, J.; Dumas, C.; Cladière, M.; Fitoussi, G.; Brun, E.; Billault, I.; Sicard-Roselli, C. CHIMACTIV: An Open-Access Website for Student-Centered Learning in Analytical Chemistry. J. Chem. Educ. 2020. https://doi.org/10.1021/acs.jchemed.0c00023.

(36) Chasteen, S. V.; Perkins, K. K.; Code, W. J.; Wieman, C. E. The Science Education Initiative: An Experiment in Scaling up Educational Improvements in a Research University. Transform. Inst. Undergrad. STEM Educ. 21st Century 2015, 125-139.

(37) Adams, W. K.; Alhadlaq, H.; Malley, C. V.; Perkins, K. K.; Olson, J.; Alshaya, F.; Alabdulkareem, S.; Wieman, C. E. Making Science Simulations and Websites Easily Translatable and Available Worldwide: Challenges and Solutions. J. Sci. Educ. Technol. 2012, $21(1), 1-10$. 
(38) Schaftenaar, G.; Noordik, J. H. Molden: A Pre-and Post-Processing Program for Molecular and Electronic Structures. J. Comput. Aided Mol. Des. 2000, 14 (2), 123-134.

(39) Humphrey, W.; Dalke, A.; Schulten, K. VMD: Visual Molecular Dynamics. J. Mol. Graph. 1996, 14 (1), 33-38. 